\title{
Molecular Communication Link
}

\author{
Nariman Farsad, Weisi Guo ${ }^{\dagger}$, Andrew Eckford \\ Dept. of Electrical Engineering and Computer Science, York University, Toronto, Canada \\ ${ }^{\dagger}$ School of Engineering, University of Warwick, United Kingdom \\ Email: nariman@cse.yorku.ca; weisi.guo@warwick.ac.uk; aeckford@yorku.ca
}

\begin{abstract}
This demonstration will present the world's first macroscale molecular communication link to reliably transmit a continuous data stream. The system modulates alcohol molecules, which are then diffused via ambient and induced air currents to carry information to a receiver. The communication distance is several meters and the propagation channel we will demonstrate consists of both free space and tunnel environments. The goal is to show that molecules can be used as an alternative to electromagnetic (EM) waves in challenging environments where EM waves do not perform well.
\end{abstract}

\section{BACKGROUND}

The transfer of data using chemicals between organisms has been observed throughout history. Most notably, Charles Darwin observed the relationship between chemical signals and mating patterns in 1871. In the 20th century, the term pheromone was coined to mean a transfer of excitement [1].

Thus far, the vast majority of nano- and molecularcommunications literature have focused on nano-scale intraand inter-cell communications [2]-[5]. There have also been a number of attempts at mimicking pheromone-based communication [6]. More recently, our team has proposed and demonstrated that molecular communications over macroscale distances is not only possible, but also reliable [7], [8]. This demonstration will for the first time show the world's first macroscale molecular communication link.

\section{TEST-BED}

The demonstration is a first-generation device that the team hopes will kick-start academic and industrial revolutions in designing molecular-based communication systems. The system is inexpensive to build, and the platform is available for sale as a modifiable and re-programmable research test-bed.

\section{A. Hardware Layout}

The communication system consists of a molecular transmitter and receiver shown in Fig. 1. The propagation channel in between is several metres of either free-space or a specific structural environment (i.e., a tunnel network).

On the transmission side, the hardware consists of: i) a user inter-face for text entry, ii) a microcontroller that converts the input text into binary sequences and then modulates the sequence on chemical signals, iii) a reservoir of chemicals, and iv) a chemical release mechanism.

On the receiver side, the hardware consists of: i) a chemical sensor, ii) a microcontroller that demodulates and decodes

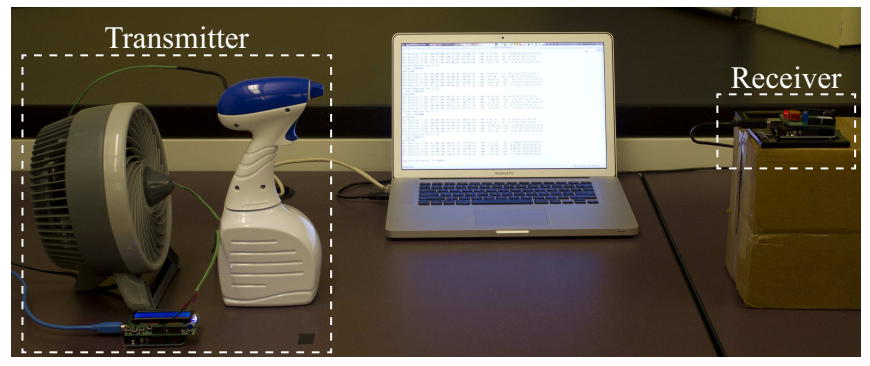

Fig. 1. The tabletop molecular communication platform.

the signal, and iii) a computer for displaying and visualizing results.

The type of data we will demonstrate is a short string of text data, because text-based information is of interest to sensor networks and command-based communication systems. For example, the team members are exploring the possibility of using molecular communications for structural health monitoring and giving commands to underground robots.

The information delivery rate for this platform is currently low because of inexpensive components and use of single type of chemical. Like the concept of bandwidth, mixtures of chemicals can clearly linearly scale the capacity. Moreover, by using more sophisticated sensors and sprays, the capacity can increase significantly.

\section{B. Health and Safety}

Due to the fact that a low volume of alcohol is diffused in open air, the demonstration will be performed behind a transparent shielded screen to respect religious sensitivities and avoid any unwanted alcohol odors in the conference venue. The alcohol used will be safe for human consumption and of a small quantity, so there is no direct chemical risk involved.

\section{Demonstration Output}

The demonstration's main goal is to show that messages can be continuously and reliably carried by modulated chemical molecules across open space. Fig. 2 shows the sample text entered at the transmitter and received at the receiver.

\section{ApPLICATION}

\section{A. Research}

In terms of research activities, existing molecular communication researchers have relied heavily on diffusion equations 


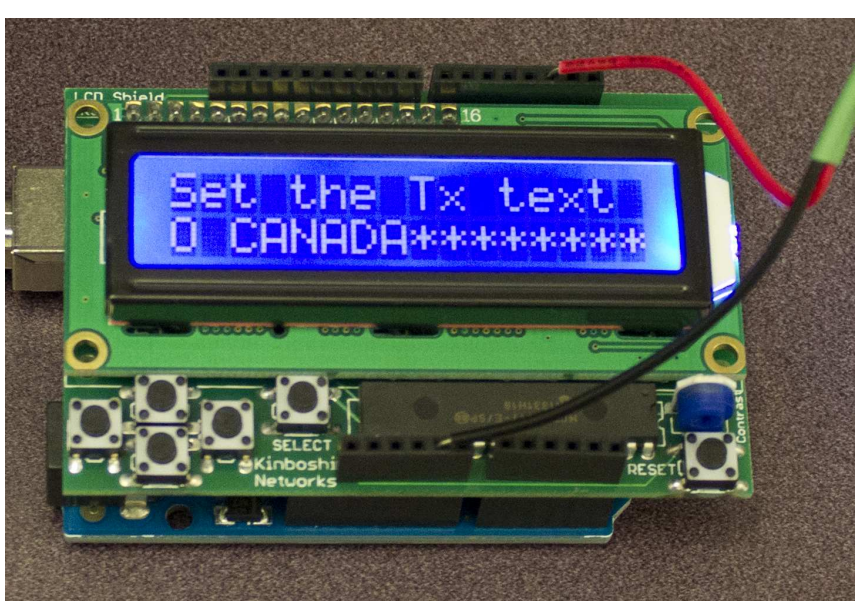

(a)

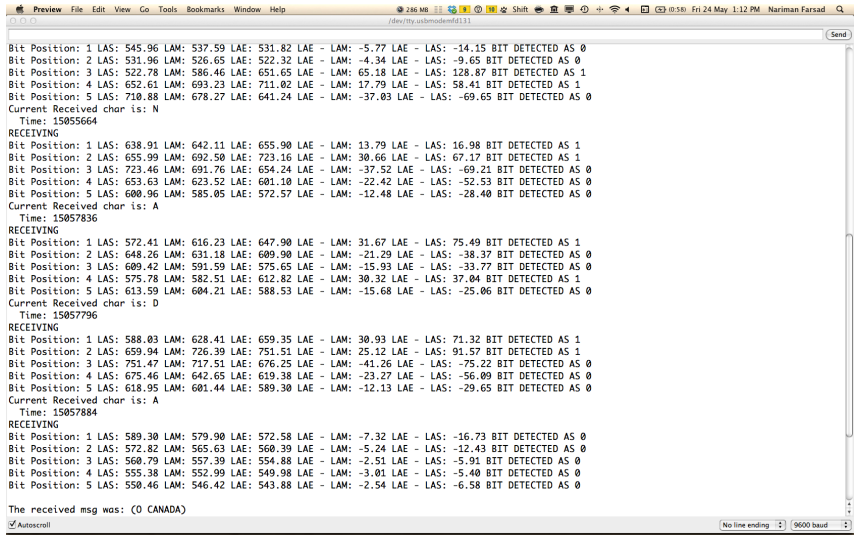

(b)

Fig. 2. Sample text message entered at the tansmitter (a), and received at the receiver (b).

and Monte-Carlo simulations to predict the performance of chemical-based data transfer. Advancing this, the test-bed provides ground truths in experimental data. For examples, most previous works [3], [9] have assumed perfect transmission, propagation and reception. However, these assumptions do not hold for this platform. Therefore, more realistic models are necessary. Some researchers are working on developing accurate mathematical models for this platform.

Another property that is observed for this platform is the fact that the end-to-end system response is nonlinear [8]. The exact cause of the nonlinearity is not known yet, and more research is required to determine and model the cause of nonlinearity. Some researchers are working of understanding and resolving the nonlinearity.

The test-bed has already received orders from 2 academic institutions and a commercial company has been setup to manage production and future research development. Moreover, the platform has initiated new research projects in these institutions. Some of these works include development of new modulation schemes to increase data rate, efforts on modelling this system and its inherent nonlinearity, and works on the next generation test-bed with more accurate components.

\section{B. Industry}

In terms of industrial interest, the test-bed's sensor network application is targeted towards structural health monitoring (smart cities) and transmitting commands to robots in subterranean areas. Existing work in tunnels has shown that EM wave propagation is difficult to predict and that communication links can often falter due to long-term structure and environment changes (i.e., wet surfaces) [10]. The project has already received funding to further develop the potential for structural health monitoring, and this demonstrator will hopefully attract further industrial interest.

\section{CONCLUSION}

In this demonstration we present the first macroscale molecular communication system that is capable of transmitting short text messages, and verify the feasibility of using chemical signals for transferring information. Our goal is to show that molecules can be used as an alternative to electromagnetic (EM) waves in challenging environments. At macroscale, the platform can be targeted towards structural health monitoring in smart cities and robotics. Moreover, the platform can be shrinked in next generations until a microscale and eventually a nanoscale molecular communication is achieved. The final goal of the demonstration is to motivate researches, and fill an important gap between theory and practice of molecular communication.

\section{Acknowledgement}

The work in this paper has been partly funded by the Royal Society, and the Warwick University Global Research Priority. Also, this work was partly funded by a Discovery grant from the Natural Sciences and Engineering Research Council (NSERC) in Canada.

\section{REFERENCES}

[1] T. D. Wyatt, "Fifty years of pheromones," in Nature, vol. 457, Jan. 2009, pp. $262-263$.

[2] B. Atakan and O. Akan, "Deterministic capacity of information flow in molecular nanonetworks," in Nano Communication Networks, Elsevier, vol. 1, Mar. 2010, pp. 31-42.

[3] K. Srinivas, A. Eckford, and R. Adve, "Molecular Communication in Fluid Media: The Additive Inverse Gaussian Noise Channel," in IEEE Transactions on Information Theory, vol. 8, Jul. 2012, pp. 4678-4692.

[4] L. Meng, P. Yeh, K. Chen, and I. Akyildiz, "MIMO communications based on molecular diffusion," in Global Communications Conference (GLOBECOM), 2012 IEEE, Dec. 2012, pp. 5380-5385.

[5] M. Leeson and M. Higgins, "Forward error correction for molecular communications," in Nano Communication Networks, Elsevier, vol. 3, 2012, pp. 161-167.

[6] M. Cole, J. Gardner, S. Pathak, T. Pearce, and Z. Rcz, "Towards a biosynthetic infochemical communication system," Procedia Chemistry, vol. 1, no. 1, pp. 305-308, 2009.

[7] W. Guo, S. Wang, A. Eckford, and J. Wu, "Reliable communication envelopes of molecular diffusion channels," in Electronics Letters, IET, vol. 49, Sep. 2013, pp. 1-2.

[8] N. Farsad, W. Guo, and A. Eckford, "Tabletop molecular communication: Text messages through chemical signals," in PLOS ONE, to appear 2013.

[9] M. Pierobon and I. F. Akyildiz, "A physical end-to-end model for molecular communication in nanonetworks," in Selected Areas in Communications, IEEE Journal on, vol. 28, 2010, pp. 602-611.

[10] F. Stajano, N. Hoult, I. Wassell, P. Bennett, C. Middleton, and K. Soga, "Smart bridges, smart tunnels: Transforming wireless sensor networks from research prototypes into robust engineering infrastructure," in $\mathrm{Ad}$ Hoc Networks, Elsevier, vol. 8, Nov. 2010, pp. 872-888. 